\title{
Biodiversidade Vegetal de uma Área do Parque Ambiental do Brejo dos Buritis em Gilbués, Piauí, Brasil
}

\section{Vegetal Biodiversity of an Area of Environment Park Brejo dos Buritis in Gilbués, Piauí, Brazil}

\author{
Raquel Soares Dias ${ }^{\mathrm{ab}} ;$ Renato Lopes Soares ${ }^{\mathrm{ab}} ;$ Rejaneide Alves Maciela ${ }^{\mathrm{ab}}$, Leudimar Aires Pereira ${ }^{\mathrm{ab}}$; \\ Polyanna Araújo Alves Bacelar*ab \\ 'Universidade Federal do Piauí, \\ bUniversidade Aberta do Brasil, PI, Brasil. \\ *E-mail: polyannabio_gen@hotmail.com
}

\begin{abstract}
Resumo
A biodiversidade da flora, o funcionamento e a conservação dos ecossistemas de ambientes degradados podem ser entendidos através de informações geradas por pesquisas florísticas. Assim, objetivou-se realizar um inventário florístico de fanerógamas do Parque Ambiental do Brejo do Buritis em Gilbués, Piauí. Para tal, foi realizado o método de ponto quadrante com quatro parcelas de $10 \times 10 \mathrm{~m}^{2}$, na região que abrange uma nascente hídrica próxima à zona urbana. O material coletado foi herborizado, identificado, classificado e as duplicatas foram direcionadas ao acervo do Herbário Graziela Barroso da Universidade Federal do Piauí (TEPB), para ratificação da classificação e registro. Concomitantemente, foram realizadas análise observacional e estudo bibliográfico para caracterização do ecossistema e identificação de fatores relacionados à vulnerabilidade ambiental. A região em estudo apresentou vegetação típica com mata ciliar e trechos de vereda, no entanto, é uma área poluída com lixo doméstico, construção de moradia irregular e desmatamento, que podem influenciar o assoreamento do brejo. Foram coletadas trinta espécies de fanerógamas distribuídas em vinte e três famílias, destas, $50 \%$ foram incluídas no herbário e estão disponíveis para estudantes e pesquisadores. As mais representativas em número de espécimes foram Piperaceae (17,7\%), Poaceae (11,1\%), Arecaceae $(6,6 \%)$, Cucurbitaceae $(6,6 \%)$ e Vitaceae $(6,6 \%)$. Foram identificadas espécies com propriedades farmacológicas, econômicas, bioindicadores, ornamentais e de uso em recuperação de áreas degradadas. Esta pesquisa possibilitou o conhecimento de uma amostra da biodiversidade vegetal do Parque Ambiental do Brejo dos Buritis, primordial para estratégias de conservação dos ecossistemas e reflorestamento, ações preconizadas pelos Objetivos do Desenvolvimento Sustentável (ODS) da agenda 2030.
\end{abstract}

Palavras-chave: Inventário Florístico. Degradação Ambiental. Unidade de Conservação.

\begin{abstract}
The biodiversity of flora, the functioning and conservation of ecosystems in degraded environments can be understood through information generated by floristic surveys. Thus, the objective was to carry out a floristic inventory of phanerogamous of Environmental Park Brejo dos Buritis in Gilbués, Piaui. Thus, the Quadrant Point method was performed with four $10 \times 10 \mathrm{~m}^{2}$ plots, in the region that covers a water source near the urban area. The collected material was herborized, identified, classified and the duplicates were directed to the collection of Graziela Barroso Herbarium of the Federal University of Piaui (UFPI), for ratification of classification and registration. Concomitantly, observational analysis and bibliographic study were performed to characterize the ecosystem and identify factors related to environmental vulnerability. The region under study presented typical vegetation with riparian forest and stretches of path, however it is a polluted area with household waste, irregular housing construction and deforestation, which can influence the swamp siltation. Thirty species of phanerogams distributed in 23 families were collected, of which 50\% were included in the herbarium and are available to students and researchers. The most representative in number of specimens were Piperaceae (17.7\%), Poaceae (11.1\%), Arecaceae (6.6\%), Cucurbitaceae (6.6\%) and Vitaceae (6.6\%). Species with pharmacological, economic, bioindicator, ornamental and recovery use of degraded areas were identified. This research allowed the knowledge of a sample of the vegetal biodiversity of the Environmental Park Brejo dos Buritis, primordial for strategies of ecosystem conservation and reforestation, actions recommended by the 2030 Agenda Sustainable Development Goals (SDG).
\end{abstract}

Keywords: Floristic Inventory. Ambiental Degradation. Conservation Unit.

\section{Introdução}

O Brasil possui uma rica variedade de espécies vegetais distribuídas em biomas com características distintas, que são a Amazônia, a Caatinga, o Cerrado, a Mata Atlântica, o Pampa e o Pantanal (ROMA, 2007). Destes biomas, apenas os Pampas e o Pantanal não fazem parte do Nordeste do país. Na regionalização do Piauí, a predominância é da Caatinga e Cerrado (FUNDAÇÃO CEPRO, 2013). A biodiversidade da flora que compõe esses ambientes é essencial para o equilíbrio dos ecossistemas e está diretamente relacionada aos fatores do micro e macroclima, tais como: a temperatura, chuva, sequestro de carbono, umidade, poluição, radiação e evapotranspiração (BURIOL et al., 2019; SHOJAEI et al., 2017; WANDERLEY; MIGUEL, 2019).

Uma forma de conhecer essa biodiversidade é por meio de levantamentos florísticos, que são técnicas primordiais para a identificação das espécies vegetais e aplicações de políticas de preservação, de recuperação e de conservação (ALVES et al., 2015; CHAVES et al., 2013; ELIAS et al., 2018). Para tal, realizar um inventário da composição vegetal é o primeiro passo para entender o funcionamento dos ecótopos e é fundamental para reconhecer ambientes degradados (FREITAS; MAGALHÃES, 2012). 
As degradações ambientais ocorrem, predominantemente, por consequência das atividades ocasionadas pela ação humana que provocam o empobrecimento do solo, podendo levar à desertificação (BRANCO et al., 2013; DIAS et al., 2016; MARIANO et al., 2018).

O Brasil possui extensas áreas tendenciosas à desertificação em nove Estados da região Nordeste, as quais afetam cerca de 1.488 municípios (FRANÇA et al., 2017). Entre esses, o Estado do Piauí apresenta ambientes de degradação na Região Sul e Sudeste (CARNEIRO et al., 2012; FERNANDES, 2012), sendo o maior núcleo de desertificação em Gilbués, ocasionado pela variabilidade climática, estrutura geológica, características de relevo, solos e vegetação (ALMEIDAFILHO; CARVALHO, 2010; SILVA, 2011; PEREZ-MARIN et al., 2012; FRANÇA et al., 2017).

O município de Gilbués tem um manancial denominado popularmente como 'Brejo', que até as décadas anteriores ao ano 2000 possuía perceptível diversidade florística, faunística, nascentes e com fluxo de água limpa e abundante. Essa área foi ocupada de forma descontrolada, com o aumento de queimadas e desmatamentos, o que acarretou em vulnerabilidade ambiental (AQUINO; OLIVEIRA; AQUINO, 2017; SANTOS et al., 2013). No entanto, considerando a importância e potencialidade desse local como uma Unidade de Conservação, houve a regularização do referido espaço para o então denominado Parque Ambiental do Brejo dos Buritis, por meio do Decreto $\mathrm{n}^{\circ}$ 031/2017 de 24 de julho (PIAUÍ, 2017).

Um inventário sobre a biodiversidade vegetal no Brejo de Gilbués é primordial para entender os padrões da natureza e o equilíbrio ambiental, facilitar o manejo de ecossistemas e sua preservação, assim como colaborar para a restauração desta área com espécies possivelmente ameaçadas (MARIANO et al., 2019). Além disso, esse tipo de ação está presente nos Objetivos do Desenvolvimento Sustentável - ODS da agenda 2030, especificamente as metas do objetivo 15 que é sobre proteção, recuperação e promoção do uso sustentável dos ecossistemas terrestres (BRASIL, 2017).

Nesse sentido, e com o intuito de ampliar as informações sobre a flora em uma área do Parque Ambiental do Brejo dos Buritis no município de Gilbués, o objetivo deste trabalho foi realizar um inventário florístico de fanerógamas para contribuir no conhecimento da biodiversidade.

\section{Material e Métodos}

O trabalho foi realizado no município de Gilbués, área de $3.495 \mathrm{~km}^{2}\left(09^{\circ} 49^{\circ} 55^{\circ}\right.$ de latitude Sul e $45^{\circ} 20^{\circ} 38^{\circ}$ de longitude Oeste), situado a Sudoeste do Piaú, na microrregião do Alto
Médio Gurguéia, distando aproximadamente $815 \mathrm{~km}$ da capital Teresina. A área específica de estudo é um manancial no Parque Ambiental do Brejo dos Buritis e se localiza transversalmente no município, na extensão dos bairros Santo Antônio, São José e Centro da cidade (Figura 1A e 1B). Inicialmente, foram realizadas pesquisas bibliográficas, análise observacional e registros fotográficos para caracterizar a região em estudo. Em seguida, para as coletas dos espécimes vegetais, foram estabelecidos locais específicos no perímetro que abrange a nascente mais próxima da área urbana para a instalação de parcelas quadradas, conforme o método quantitativo por parcelas preconizado por Muller-Dombois e Ellenberg (1974). Nesse local, foram instalados, ao acaso, quatro parcelas de $10 \times 10 \mathrm{~m}^{2}\left(100 \mathrm{~m}^{2}\right)$, totalizando uma área amostral de $400 \mathrm{~m}^{2}$, com intervalos de trinta metros de distância entre as parcelas (Figura 1C). Foram coletados todos os espécimes de fanerógamas presentes nas parcelas, independente do porte (árvore, arbustivo, subarbustivo, herbáceo e liana/cipó) ou estágio fenológico. As coletas de fanerógamas foram realizadas quinzenalmente no período de Agosto a Setembro de 2017.

Figura 1 - Mapa evidenciando o município de Gilbués, Piauí, Brasil (A). Cobertura arbórea densa é o Brejo do município e o círculo em amarelo indica a área definida para coleta (B). Organização espacial das parcelas (C).

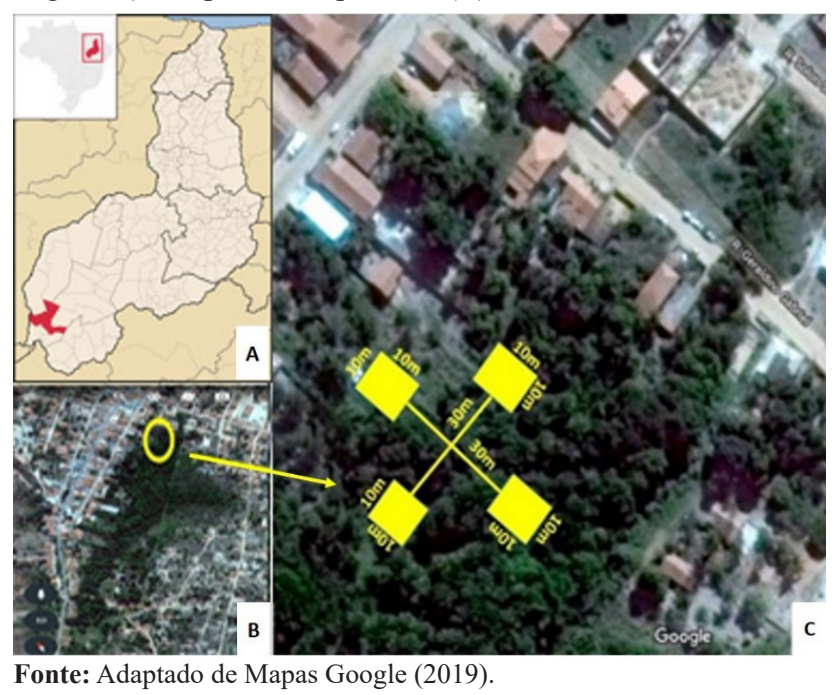

Dentro de cada parcela foram coletadas cinco amostras de todos os espécimes de fanerógamas (Figura 2A). Os exemplares foram acondicionados em sacos plásticos devidamente identificados com os dados de coleta de campo (Figura 2B). Em seguida, as amostras foram encaminhadas ao laboratório do polo de Ensino a Distância 'Chapada das Mangabeiras' da Universidade Aberta do Brasil, para serem herborizados (Figura $2 \mathrm{C}$ e D). 
Figura 2 - Coleta de campo e acondicionamento (A e B). Herborização das fanerógamas coletadas (C e D), coletadas no Parque Ambiental do Brejo dos Buritis/ Brejo de Gilbués, Gilbués-Brasil, 2017

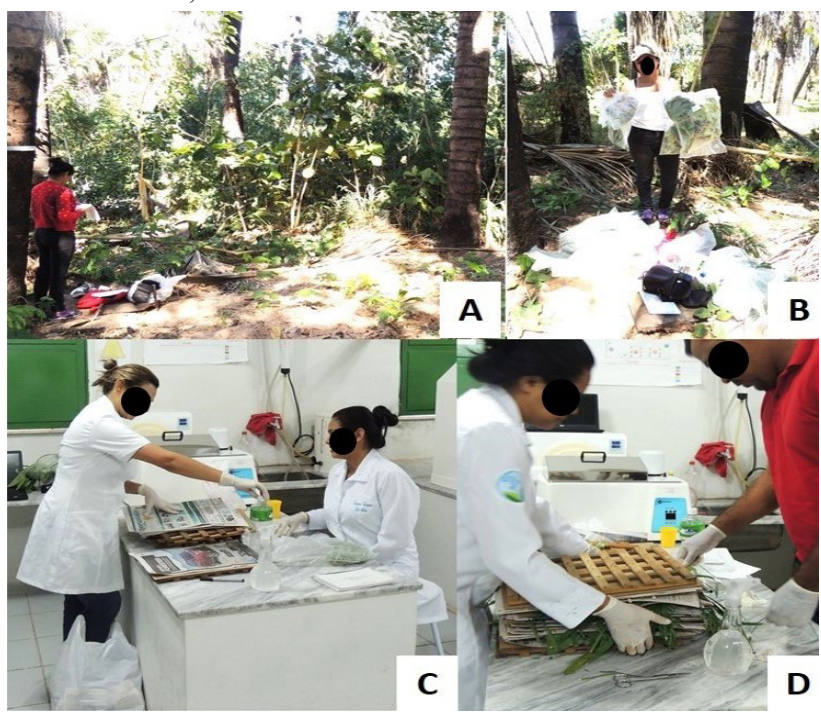

Fonte: Os autores.

Os espécimes foram identificados com o auxílio do Herbário Virtual Reflora. O sistema de classificação utilizado foi do “Angiosperm Plylogeny Group”APG III (2009). As informações das amostras coletadas foram tabuladas e o número de indivíduos encontrados foram organizados para demonstrar a representatividade por família. Foram realizados os registros fotográficos dos espécimes coletados, com o uso de câmera digital Nikon Coolpix P510 de 16.1 megapixels, e as imagens foram organizadas e editadas no brilho e contraste pelo software Adobe Photoshop CS3. As duplicatas foram direcionadas ao acervo do Herbário Graziela Maciel Barroso (acrônimo TEPB), pertencente à Universidade Federal do Piauí.

\section{Resultados e Discussão}

Na observação de campo, foi constatado no Parque Ambiental do Brejo dos Buritis (Brejo de Gilbués) a presença de vegetação com característica específica com trechos de veredas compostos por mata ciliar, solo hidro mórfico e grande densidade de espécimes de buritis (Mauritia flexuosa L. f.), assim como reportaram Reis, Silva e Ribeiro (2016), em estudo realizado na região.

No entanto, observou-se a presença de uma quantidade excessiva de lixo doméstico descartado irregularmente, bem como construção de moradias irregulares que contribuem, significativamente, para o aceleramento do processo de degradação do leito do brejo (Figura 3). Estes achados também foram relatados em estudo feito por Reis, Silva e Ribeiro (2016), que descreveram o Brejo em uma situação de estágio acelerado de degradação, de poluição, de assoreamento, de desmatamento, de presença de queimadas e moradias irregulares nas margens. Esses fatores favorecem o surgimento e ampliação dos solos erodidos, que implicam no soterramento dos brejos e leitos dos rios (SILVA, 2011).

Figura 3 - Área do Parque Ambiental do Brejo dos Buritis com descarte de lixo doméstico (A), assoreamento (B) e córrego do brejo degradado (C). Seta vermelha em A indica domicílio próximo

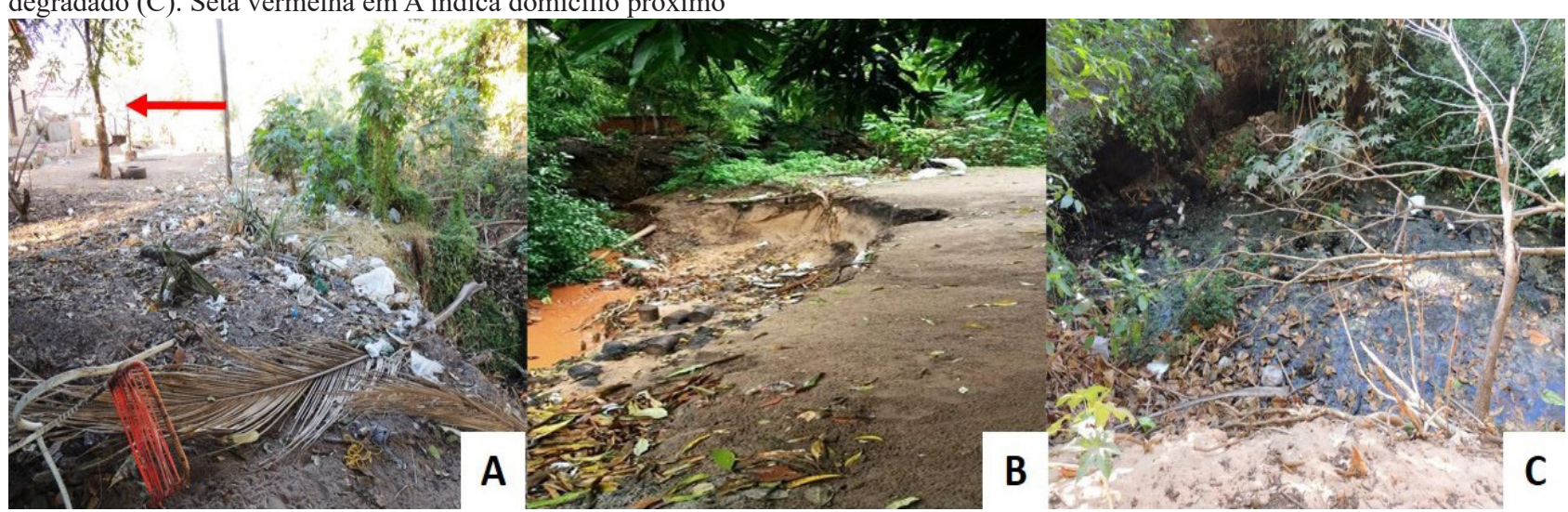

Fonte: Dados da pesquisa.

Conforme apresentado na Figura 3A, o volume de lixo doméstico descartado na margem do brejo indica que a urbanização desordenada nas mediações do parque é um forte indício de ser a principal causa da vulnerabilidade nesse local e pode ter acelerado o processo de degradação ambiental na área (FRANÇA et al., 2017; COSTA; VIEIRA, 2018). Entretanto, atividades como as reportadas por Patrício, Silva e Ramos (2012), em trabalho chamando a atenção para o desenvolvimento de ações no controle da vulnerabilidade ambiental, nestas áreas em Gilbués, tem minimizado a degradação ambiental, melhorando o combate ao agravo da desertificação e possibilitando a conservação da biodiversidade.

Vale ressaltar que esta área é bastante afetada por impactos provenientes da proximidade ao Núcleo de Desertificação de Gilbués. Porém, ações de recuperação estão sendo implantadas para diminuir os danos ambientais, como a relatada por Costa e Vieira (2018) e as realizadas pelo Núcleo de Pesquisa de Recuperação de Áreas Degradadas e Combate à Desertificação (NUPERADE). 
Sobre a aquisição de material florístico para o inventário de fanerógamas da nascente do Parque Ambiental do Brejo dos Buritis, foi possível coletar 45 amostras, sendo 14 da primeira parcela, 09 da segunda, 11 da terceira e 11 da quarta. Deste material, 30 espécies foram identificadas e distribuídas em 23 famílias (Quadro 1 e Figura 4).

Quadro 1 - Família, espécie, número da coleta $\left(\mathrm{N}^{\circ}\right)$ e registro no Herbário Graziela Maciel Barroso das espécies vegetais coletadas na nascente do Parque Ambiental do Brejo dos Buritis, Gilbués, Piauí, Brasil, 2017

\begin{tabular}{|c|c|c|c|}
\hline Família & Espécie & $\mathbf{N}^{0}$ & Registro \\
\hline Acanthaceae & $\begin{array}{l}\text { Odontonema cuspidatum } \\
\text { (Nees) Kuntze }\end{array}$ & 01 & 31569 \\
\hline Amaranthaceae & $\begin{array}{l}\text { Gomphrena celosioides } \\
\text { Mart. }\end{array}$ & 02 & 31570 \\
\hline Apocynaceae & $\begin{array}{l}\text { Himatanthus bracteatus (A. } \\
\text { DC.) Woodson }\end{array}$ & 03 & - \\
\hline \multirow[b]{2}{*}{ Araceae } & $\begin{array}{c}\text { Dieffenbachia seguine } \\
\text { (Jacq.) Schott }\end{array}$ & 04 & - \\
\hline & $\begin{array}{c}\text { Philodendron } \\
\text { appendiculatum Nadruz \& } \\
\text { Mayo }\end{array}$ & 05 & 31574 \\
\hline Arecaceae & Mauritia flexuosa L. f. & 06 & - \\
\hline \multirow[b]{2}{*}{ Asteraceae } & Bidens pilosa $\mathrm{L}$. & 07 & 31576 \\
\hline & $\begin{array}{l}\text { Vernonia polyanthes } \\
\text { (Spreng.) Less. }\end{array}$ & 08 & 31577 \\
\hline Caricaceae & Carica papaya $\mathrm{L}$. & 09 & - \\
\hline Chysobalanaceae & $\begin{array}{c}\text { Licania tomentosa (Benth.) } \\
\text { Fritsch }\end{array}$ & 10 & 31578 \\
\hline Combretaceae & Terminalia catappa $\mathrm{L}$. & 11 & - \\
\hline Commelinaceae & Commelina benghalensis L. & 12 & - \\
\hline \multirow{2}{*}{ Cucurbitaceae } & $\underline{\text { Cucumis } s p \mathrm{~L} .}$ & 13 & - \\
\hline & Momordica charantia $\mathrm{L}$. & 14 & 31579 \\
\hline Cyperaceae & Cyperus involucratus Rottb. & 15 & 31580 \\
\hline Euphorbiaceae & Ricinus communis L. & 16 & 31581 \\
\hline Fabaceae & $\begin{array}{c}\text { Leucaena leucocephala } \\
\text { (Lam.) de Wit }\end{array}$ & 17 & - \\
\hline Lamiaceae & Prunella vulgaris $\mathbf{L}$. & 18 & 31582 \\
\hline Malvaceae & Guazuma ulmifolia Lam. & 19 & - \\
\hline Myrtaceae & Syzygium cumini (L.) Skeels & 20 & - \\
\hline \multirow{2}{*}{ Piperaceae } & Piper aduncum L. & 21 & 31583 \\
\hline & Piper amplum Kunth & 22 & - \\
\hline \multirow{3}{*}{ Poaceae } & $\begin{array}{c}\text { Lasiacis ligulata Hitchc. \& } \\
\text { Chase }\end{array}$ & 23 & 31584 \\
\hline & $\begin{array}{l}\text { Pennisetum purpureum } \\
\text { Schumach. }\end{array}$ & 24 & - \\
\hline & Merostachys sp Spreng. & 25 & - \\
\hline Rubiaceae & Genipa $s p \mathrm{~L}$. & 26 & - \\
\hline Turneraceae & Turnera ulmifolia $\mathrm{L}$. & 27 & 31585 \\
\hline \multirow[b]{2}{*}{ Urticaceae } & Parietaria lusitanica $\mathrm{L}$. & 28 & 31586 \\
\hline & $\begin{array}{l}\text { Cecropia pachystachya } \\
\text { Trécul } \\
\end{array}$ & 29 & - \\
\hline Vitaceae & $\begin{array}{l}\text { Cissus verticillata } \text { (L.) } \\
\text { Nicolson \& C.E.Jarvis }\end{array}$ & 30 & 31587 \\
\hline
\end{tabular}

Fonte: Dados da pesquisa.
Figura 4 - Espécies Odontonema cuspidatum (01), Gomphrena celosioides (02), Himatanthus bracteatus (03), Dieffenbachia seguine (04), Philodendron appendiculatum (05), Mauritia flexuosa (06), Bidens pilosa (07), Vernonia polyanthes (08), Carica papaya (09), Licania tomentosa (10), Terminalia catappa (11), Commelina benghalensis (12), Cucumis sp (13), Momordica charantia (14), Cyperus involucratus (15), Ricinus communis (16), Leucaena leucocephala (17), Prunella vulgaris (18), Guazuma ulmifolia (19), Syzygium cumini (20), Piper aduncum (21), Piper amplum (22), Lasiacis ligulata (23), Pennisetum purpureum (24), Merostachys sp (25), Genipa sp (26), Turnera ulmifolia (27), Parietaria lusitanica (28), Cecropia pachystachya (29) e Cissus verticillata (30) coletadas no Parque Ambiental do Brejo dos Buritis, Gilbués, Piauí, Brasil, 2017.

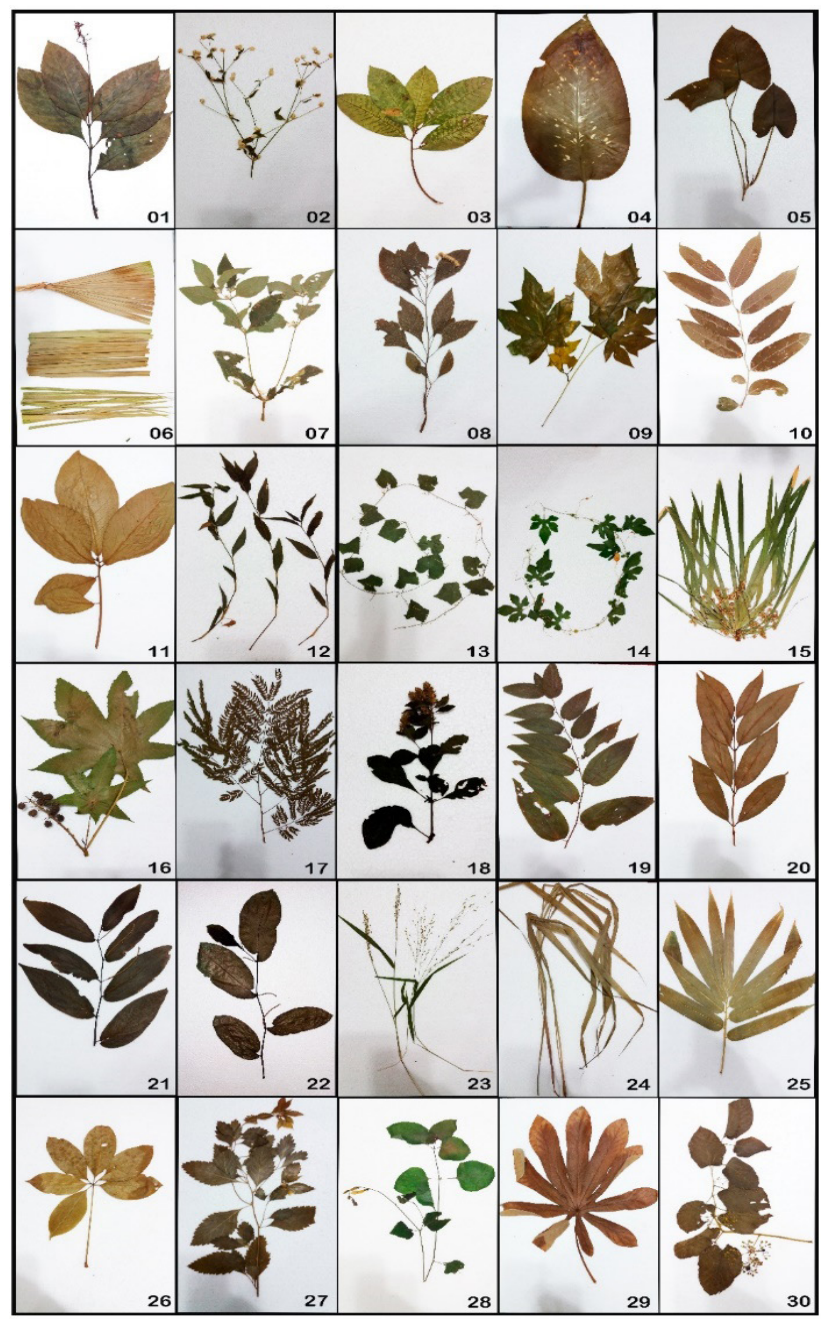

Fonte: Dados da pesquisa.

Levando em consideração os hábitos das plantas, verificou-se que das 30 espécies identificadas, 33,33\% (10/30) eram árvores, $10 \%(3 / 30)$ arbustivas, 6,67\% (2/30) subarbustivas, $43,33 \%$ (13/30) herbáceas e $6,67 \%(2 / 30)$ eram liana/trepadeiras. As famílias mais representativas em número de indivíduo (espécimem) foram Piperaceae $(17,7 \%, 8 / 45)$, Poaceae $(11,1 \%, 5 / 45)$, Arecaceae $(6,6 \%$, $3 / 45)$, Cucurbitaceae $(6,6 \%, 3 / 45)$ e Vitaceae $(6,6 \%, 3 / 45)$. As famílias Euphorbiaceae, Fabaceae, Asteraceae, Urticacea e Combreaceae com $4,4 \%$ e as demais apenas com 2,2\% (2/45) (Quadro 1).

A família botânica Piperacea foi coletada em todas as 
parcelas, com duas espécies identificadas do gênero Piper sp., comum em área úmida ou borda de mananciais, assim como P. amplum que foi coletada às margens do brejo. Essa espécie, além de essencial para o equilíbrio ecológico da região, é relatada com extrema importância na literatura por possuir propriedades biológicas e princípios ativos importantes para a área da farmacologia (PERIGO et al., 2016), assim como O. cuspidatum (REFAEY et al., 2015), G. celosioides (OLUWABUNMI; ABIOLA, 2015), B. pilosa (DEBA et al., 2008), V. polyanthes (NASCIMENTO et al., 2015), T. catappa (ANAND; DIVYA; KOTTI, 2015), M. charantia (JOSEPH; JINI, 2013), P. vulgaris (ZHANG; HE; HU, 2011), S. cumini (BANDIOLA et al., 2017), T. ulmifolia (ALMEIDA; ALVES; AMARAL, 2012), P. lusitanica (ARCUS et al., 2011) e $C$. pachystachya (ARAGÃO et al., 2013).

Outras espécies, além de propriedades farmacológicas, possuem potencial ornamental e de recuperação em áreas degradadas como H. bracteatus (PUGAS; PAZ; NEVES, 2018); apresentam interesse econômico por serem utilizadas na alimentação, no artesanato e na confecção de móveis como M. flexuosa (KOOLEN et al., 2013); é bioindicador e apresenta potencialidade no reflorestamento como $L$. tomentosa (MAIOLI et al., 2008; PESSOA et al., 2016). Assim, ficou evidenciada a importância da preservação e da conservação destas espécies.

As duplicatas dessas espécies foram encaminhadas ao Herbário Graziela Maciel Barroso, da Universidade Federal do Piauí, das quais 50\% foram incorporadas ao acervo, porém as demais não foram incorporadas por necessitar de mais estruturas de floração e frutos, para ratificação. Esse processo de inclusão e registro em herbário foi relevante para que as informações sobre a biodiversidade florística do ambiente em questão fiquem disponíveis como material didático e científico para estudantes e pesquisadores, bem como para auxiliar em estratégias de conservação e de preservação (BARROS; ABREU, 2015; FONSECA; VIEIRA, 2015). Estes dados se tornam mais acessíveis visto que os herbários realizam intercâmbios e que a informatização viabiliza a permuta rápida de informações (DA COSTA et al., 2016).

Em relação ao quantitativo, o número de espécies encontradas foi pequeno, indicando a hipótese de que o desmatamento e a degradação ambiental presentes na região propiciaram o assoreamento da margem do brejo e provocaram a perda de alguns representantes vegetais. Entretanto, esta pesquisa foi significativa por possibilitar a inferência sobre algumas das principais espécies vegetais essenciais para manutenção da biodiversidade do Parque Ambiental do Brejo dos Buritis, que consequentemente contribuiu com as metas 1,2 e 3 dos ODS, que se referem à conservação, recuperação, sustentabilidade e restauração de ambientes degradados como o do presente estudo (BRASIL, 2017).

Verificou-se que o brejo possui relevância ecológica e regional por constituir refúgios fauno-florísticos e por ser nascedouro das fontes hídricas, similar a relato de estudos no mesmo ambiente no Espírito Santo e em um brejo de altitude no Pernambuco (PEREIRA; SILVA; BARBOSA, 2010; VALADARES et al., 2011).

\section{Conclusão}

Gilbués, por ter extensas áreas vulneráveis pela existência e proximidade com o núcleo de desertificação, se torna uma região com extrema necessidade de políticas públicas que possibilitem a conservação de sua biodiversidade e que garanta a preservação dos ecossistemas. Neste município, a região do Brejo que foi consolidada como Parque Ambiental do Brejo dos Buritis apresentou sério processo de degradação ambiental, com acúmulo de lixo doméstico na nascente estudada, desmatamento, assoreamento, presença de queimadas e construção de moradia irregular próximo à margem.

O inventário florístico possibilitou o conhecimento de uma amostra da biodiversidade vegetal do parque e foram identificadas espécies com propriedades farmacológicas, potencial econômico, ornamental, uso em reflorestamento e como bioindicador. As amostras inclusas no acervo do Herbário Graziela Barroso (TEPB) ficarão disponíveis para serem acessadas por quaisquer estudantes e pesquisadores.

Além disso, os dados obtidos servirão de subsídios para estratégias complementares de revitalização e de recuperação florestal neste ambiente, assim como será fundamental para expandir informações sobre a importância da preservação das espécies vegetais para conservação de área úmida, brejo e riacho, o que também contempla o objetivo 15 dos ODS, da agenda 2030 .

\section{Agradecimentos}

Ao Herbário Graziela Maciel Barroso (TEPB) por gentilmente proporcionar suporte às coletas e receber as duplicatas para inserção no acervo.

\section{Referências}

ALMEIDA, F.M.D.; ALVES, M.T.S.S. D.; AMARAL, F.M.M.D. Uso de plantas com finalidade medicinal por pessoas vivendo com HIV/AIDS em terapia antirretroviral. Saúde e Sociedade, v.21, n.2, p.424-434, 2012.

ALMEIDA-FILHO, R.; CARVALHO, C.M. Mapping land degradation in the Gilbues region, northeastern Brazil, using Landsat TMimages. International Journal of Remote Sensing, v.31, n.4, p.1087-1094, 2010. doi: 10.1080/01431160903260957

ALVES, M. et al. Levantamento florístico de um remanescente de Mata Atlântica no litoral norte do estado da Bahia, Brasil. Hoehnea, v.42, n.3, p.581-595, 2015. doi: 10.1590/2236-8906$06 / 2015$

ANAND, A.V.; DIVYA, N.; KOTTI, P.P. An updated review of Terminalia catappa. Pharmacognosy reviews, v.9, n.18, p.93-98, 2015.

Angiosperm Phylogeny Group (APG). An update of the Angiosperm Phylogeny Group APG III. Botanical Journal of the Linnean Society, v.161, p.105-121, 2009. 
AQUINO, C.M.S.; OLIVEIRA, J.G.B.; AQUINO, R.P. Erosividade das Chuvas do Município de Gilbués, Piauí, Brasil. In: PEREZ FILHO, A.; AMORIM, R.R. Os Desafios da Geografia Física na Fronteira do Conhecimento. Campinas: Instituto de geociências - UNICAMP, p.1469-1477, 2017.

ARAGÃO, D.M.O. et al. Anti-inflammatory, antinociceptive and cytotoxic effects of the methanol extract of Cecropia pachystachya Trécul. Phytotherapy Research, v.27, n.6, p.926930, 2013.

ARCUS, M. et al. Facts regarding the pharmacognostic and phytochemical study of the plant Parietaria Lusitanica L. Scientific Study \& Research. Chemistry \& Chemical Engineering, Biotechnology, Food Industry, v.12, n.2, 141, 2011.

BANDIOLA, T.M. et al. Syzygium cumini (L.) Skeels: a review of its phytochemical constituents, toxicity studies, and traditional and pharmacological uses. Int. J. Appl. Pharm. Biol. Res, v.2, n.6, p.15-23, 2017.

BARROS, R.F.M.; ABREU, J.C.O. Herbário Graziela Barroso, Piauí (TEPB). Unisanta BioScience, v.4, n.6, p.165-168, 2015.

BRANCO, S.B.C. et al. Influence of topography and erodibility in chemical properties in a degraded area of Gilbués, PIBrazil. Revista Brasileira de Ciências Agrárias (Agrária), v.8, n.2, p.324-330, 2013. doi: 10.5039/agraria.v8i2a2418

BRASIL. Relatório nacional voluntário sobre os Objetivos de Desenvolvimento Sustentável. Secretaria de Governo da Presidência da República, Ministério do Planejamento, Desenvolvimento e Gestão - Brasília: Presidência da República, 76 p., 2017.

BURIOL, G.A. et al. Relação da vegetação natural do Estado do Rio Grande do Sul com as disponibilidades climáticas. Ciência Florestal, v.29, n.1, p.233-242, 2019. doi: $10.5902 / 1980509812382$

CARNEIRO, R.F.V. et al. Fungos micorrízicos arbusculares como indicadores da recuperação de áreas degradadas no Nordeste do Brasil. Revista Ciência Agronômica, v.43, n.4, p.648-657, 2012.

CHAVES, A.C.G. et al. A Importância dos levantamentos florístico e fitossociológico para a conservação e preservação das florestas. Agropecuária Científica no Semiárido, ACSA, v.9, n.2, p.43-48, 2013.

COSTA, G.J.A.; VIEIRA, C.I.P. Geotecnologias para análise da vulnerabilidade ambiental do núcleo de desertificação do Piauí. Cad. Prudentino de Geografia, v.2, n.40, p.59-76, 2018.

DA COSTA, J.C.M. et al. Herbário virtual e universidade: biodiversidade vegetal para ensino, pesquisa e extensão. Scie. Plena, v.12, n.6, 2016.

DEBA, F. et al. Chemical composition and antioxidant, antibacterial and antifungal activities of the essential oils from Bidens pilosa Linn. var. Radiata. Food Control., v.19, n.4, p.346352, 2008.

DIAS, L.C. et al. Patterns of land use, extensification, and intensification of Brazilian agriculture. Global Change Biol., v.22, n.8, p.2887-2903, 2016.

ELIAS, G.A. et al. Árvores de um fragmento florestal urbano em Santa Catarina, Sul do brasil: florística e estrutura. Ciênc. Florestal, v.28, n.4, p.1755-1769, 2018. doi: 10.5902/1980509835335

FERNANDES, A. Conexões Florísticas do Brasil. Fortaleza: Banco do Nordeste, 2012.

FONSECA, R.S.; VIEIRA, M.F. Coleções botânicas com enfoque em herbário. Série Conhecimento, v. 29, 2015.

FRANÇA, L.C.; PIUZANA, D.; ROSS, J.L. Fragilidade
Ambiental Potencial e Emergente em núcleo de desertificação no semiárido brasileiro (Gilbués, Piauí). Rev. Espacios (Caracas), v.38, n.31, 2017.

FREITAS, W.K.; MAGALHÃES, L.M.S. Métodos e parâmetros para estudo da vegetação com ênfase no estrato arbóreo. Floresta e Ambiente, v.19, n.4, p.520-540, 2012.

FUNDAÇÃO CEPRO. Piauí em números: situação socioeconômica - Piauí. 2013. Disponível em: http://www.cepro. pi.gov.br/download/201310/CEPRO13_aab5263f9a.pdf

JOSEPH, B.; JINI, D. Antidiabetic effects of Momordica charantia (bitter melon) and its medicinal potency. Asian Pacific J. Trop.l Dis., v.3, n.2, p.93-102, 2013.

KOOLEN, H.H. et al. Antioxidant, antimicrobial activities and characterization of phenolic compounds from buriti (Mauritia flexuosa L. f.) by UPLC-ESI-MS/MS. Food Res. Int., v.51, n.2, p.467-473, 2013

MAIOLI, O.L.G. et al. Parâmetros bioquímicos foliares das espécies Licania tomentosa (benth.) e Bauhinia forficata (link.) para avaliação da qualidade do ar. Quim. Nova, v.31, n.8, p.19251932, 2008.

MARIANO, D.A. et al. Use of remote sensing indicators to assess effects of drought and human-induced land degradation on ecosystem health in Northeastern Brazil. Remote Sensing Environ., v.213, p.129-143, 2018. doi: 10.1016/j.rse.2018.04.048

MARIANO, R.F. et al. Well-sampled regions risk losing key biological data: a case study in the Atlantic Forest. Biodiversity and Conservation, v.28, p.2581-2598, 2019. doi: 10.1007/ s10531-019-01780-1

MUELLER-DOMBOIS, D.; ELLENBERG, H. Aims and methods of vegetation ecology. New York: John Wiley \& Sons; 1974.

NASCIMENTO, A.M. et al. Antileishmanial activity of compounds produced by endophytic fungi derived from medicinal plant Vernonia polyanthes and their potential as source of bioactive substances. World J. Microbiol. Biotechnol., v.31, n.11, p.1793-1800, 2015.

OLUWABUNMI, I.J.; ABIOLA, T. Gastroprotective effect of methanolic extract of Gomphrena celosioides on indomethacin induced gastric ulcer in Wistar albino rats. Int. J. Appl. Basic Med. Res., v.5, n.1, p.41-45, 2015. doi: 10.4103/2229-516X.149238.

PATRÍCIO, M.D.C.M.; SILVA, V.M.D.A.; RAMOS, A.R.D. Gilbués - núcleo de desertificação do Piauí, caracterização física, variabilidade climática e impactos ambientais. Polêm! CA, v.11, n.3, p.470-482, 2012.

PEREIRA, R.D.C.A.; SILVA, J.A.; BARBOSA, J.I.S. Flora de um "brejo de altitude" de Pernambuco: Reserva Ecológica da Serra Negra. Anais da Academia Pernambucana de Ciência Agronômica, v.7, p.286-304, 2010.

PEREZ-MARIN, A.M. et al. Núcleos de desertificação no semiárido brasileiro: ocorrência natural ou antrópica? Parcerias Estratégicas, v.17, n.34, p.87-106, 2012.

PERIGO, C.V. et al. The chemical composition and antibacterial activity of eleven Piper species from distinct rainforest areas in Southeastern Brazil. Industrial Crops Products, v.94, p.528-539, 2016.

PESSOA, I.P. et al. Polyphenol composition, antioxidant activity and cytotoxicity of seeds from two underexploited wild Licania species: L. rigida and L. tomentosa. Molecules, v.21, n.1755, p.116, 2016.

PIAUÍ. Decreto $n^{\circ}$ 031/2017 de 26 de Julho de 2017. Diário Oficial dos Municípios, v.15, p.42-43, 2017. 
PUGAS, R.M.et al. Himatanthus bracteatus (A. DC.) Woodson (Apocynaceae): phenology, morphology, and floral biology of a species endemic to the Atlantic Forest. Hoehnea, v.45, n.3, p.405412, 2018. doi: https://doi.org/10.1590/2236-8906-23/2017

REFAEY, M.S. et al. Hepatoprotective and antioxidant activity of Odontonema cuspidatum (Nees) Kuntze against CCl4-induced hepatic injury in rats. J. Pharm. Phytochem., v.4, n.2, p.89-96, 2015.

REIS, M.P.; SILVA, M.P.; RIBEIRO, L.A. Urbanização e impactos ambientais sobre o brejo no município de Gilbués, Piauí. In: CONGRESSO BRASILEIRO DE GESTÃO AMBIENTAL, 7. Campina Grande - PB. Campina Grande: IBEAS, 2016.

ROMA, J.C. Mapa de cobertura vegetal dos biomas brasileiros. Brasil: Ministério do Meio Ambiente, 2007.

SANTOS, H.L. et al. Erosion factors and magnetic susceptibility in different compartments of a slope in Gilbués-PI, Brazil. Eng. Agríc., v.33, n.1, p.64-74, 2013.

SHOJAEI, P. et al. Effect of different land cover/use types on canopy layer air temperature in an urban area with a dry climate. Building Environ., v.125, p.451-463, 2017. doi: 10.1016/j. buildenv.2017.09.010

SILVA, I.S. Estudo da desertificação em Gilbués-Piauí: caracterização física, variabilidade climática e impactos ambientais. Rev. Geografia (Recife), v.28, n.2, p.95-108, 2011.

VALADARES, R.T. et al. Levantamento florístico de um brejoherbáceo localizado na restinga de Morada do Sol, município de Vila Velha, Espírito Santo, Brasil. Rodriguésia, v.62, n.4, p.827834, 2011.

WANDERLEY, H.S.; MIGUEL, V.C. Mudança dos elementos meteorológicos em função da degradação da floresta urbana. Ciênc. Florestal, v.29, n.2, p.834-843, 2019. doi: 10.5902/1980509832090

ZHANG, G.; HE, L.; HU, M. Optimized ultrasonic-assisted extraction of flavonoids from Prunella vulgaris L. and evaluation of antioxidant activities in vitro. Innovative Food Scie. Emerging Technol., v.12, n.1, p.18-25, 2011. 